\title{
Constraining non-linear dynamo models using quasi-biennial oscillations from sunspot area data
}

\author{
F. Inceoglu ${ }^{1,2,3}$, R. Simoniello ${ }^{4}$, R. Arlt ${ }^{5}$, and M. Rempel ${ }^{6}$ \\ 1 Department of Engineering, Aarhus University, Ny Munkegade 120, 8000 Aarhus C, Denmark \\ e-mail: fadil@eng.au.dk, fadilinceoglu@hotmail.com \\ 2 Department of Geoscience, Aarhus University, Høegh-Guldbergs Gade 2, 8000 Aarhus C, Denmark \\ 3 Stellar Astrophysics Centre, Department of Physics and Astronomy, Aarhus University, Ny Munkegade 120, \\ 8000 Aarhus C, Denmark \\ ${ }^{4}$ Geneva Observatory, University of Geneva, Geneva, Switzerland \\ 5 Leibniz-Institut für Astrophysik Potsdam, An der Sternwarte 16, 14482 Potsdam, Germany \\ ${ }^{6}$ High Altitude Observatory, National Center for Atmospheric Research, Boulder, PO Box 3000, Boulder, CO 80307, USA
}

Received 14 February 2019 / Accepted 7 April 2019

\begin{abstract}
Context. Solar magnetic activity exhibits variations with periods between 1.5 and 4 years, the so-called quasi-biennial oscillations (QBOs), in addition to the well-known 11-year Schwabe cycles. Solar dynamo is thought to be the mechanism responsible for the generation of QBOs.

Aims. In this work, we analyse sunspot areas to investigate the spatial and temporal behaviour of the QBO signal and study the physical mechanisms responsible using simulations from fully non-linear mean-field flux-transport dynamos.

Methods. We investigated the behaviour of the QBOs in the sunspot area data for the full disk, and the northern and southern hemispheres, using wavelet and Fourier analyses. We also ran solar dynamos with two different approaches to generating a poloidal field from an existing toroidal field, namely Babcock-Leighton and turbulent $\alpha$ mechanisms. We then studied the simulated magnetic field strengths as well as meridional circulation and differential rotation rates using the same methods.

Results. The results from the sunspot areas show that the QBOs are present in the full disk and hemispheric sunspot areas. These QBOs show slightly different spatial and temporal behaviours, indicating slightly decoupled solar hemispheres. The QBO signal is generally intermittent and in-phase with the sunspot area data, surfacing when the solar activity is at its maximum. The results from the BLdynamos show that they are neither capable of generating the slightly decoupled behaviour of solar hemispheres nor can they generate QBO-like signals. The turbulent $\alpha$-dynamos on the other hand generated decoupled hemispheres and some QBO-like shorter cycles. Conclusions. In conclusion, our simulations show that the turbulent $\alpha$-dynamos with the Lorentz force seem more efficient in generating the observed temporal and spatial behaviour of the QBO signal compared with the BL-dynamos.
\end{abstract}

Key words. dynamo - sunspots - Sun: activity - Sun: oscillations

\section{Introduction}

As a magnetically active star, the Sun shows cyclic variations in its activity levels. The best known of these variations is the 11-year sunspot cycle (Schwabe 1844), which is superimposed on longer-term variations such as the $\sim 90$-year Gleissberg cycle (Gleissberg 1939) and the 210 -year Suess cycle (Suess 1980). In addition to these long-term cyclic variations, the Sun also shows variations in its activity, with periods longer than a solar rotation but considerably shorter than the Schwabe cycle, such as the period of 8-11 months in the Ca-K plage index, the 150-day period related to the strong magnetic fields on the Sun (Pap et al. 1990), and the quasi-biennial oscillations (QBOs). The QBOs can be identified across from the subsurface layers (Simoniello et al. 2012, 2013) to the surface of the Sun (Benevolenskaya 1998; Vecchio et al. 2012) and to neutron counting rates measured on Earth (Kudela et al. 2010; Vecchio et al. 2010). The QBOs are therefore believed to be a global phenomenon extending from the subsurface layers of the Sun to the Earth via the open solar magnetic field.

The period of the QBO signal in solar activity indices ranges from 1.5 to 4 years (Vecchio \& Carbone 2009), while its amplitude is in phase with the Schwabe cycle. The QBOs were observed to be an intermittent signal. They attain their highest amplitude during solar cycle maxima and become weaker during solar cycle minima. The QBOs also develop independently in the solar hemispheres (Bazilevskaya et al. 2014). Based on NSO/Kitt Peak magnetic synoptic maps, Vecchio et al. (2012) suggested that the QBOs are distributed equally over all latitudes and are associated with poleward magnetic flux transportation from lower solar latitudes during the maximum of a solar cycle and its descending phase. These authors also suggested that they found an equator-ward drift at an approximately two-year rate in the radial and east-west components of the magnetic field, which was calculated using the magnetic synoptic maps.

There are several physical mechanisms proposed that could cause the observed QBOs; flip-flop cycles, which are defined as the $180^{\circ}$ shift of the active longitudes with the largest active regions (Berdyugina \& Usoskin 2003), spatiotemporal fragmentation from differences in temporal variations in the radial profile of the rotation rates (Simoniello et al. 2013), instability of magnetic Rossby waves in the tachocline (Zaqarashvili et al. 2010), and tachocline non-linear oscillations, where periodically varying energy exchange takes place between the Rossby waves, 
differential rotation, and the present toroidal field (Dikpati et al. 2018). Additionally, a secondary dynamo working in the subsurface layers has been proposed as the mechanism behind the QBOs (Benevolenskaya 1998; Fletcher et al. 2010), as the helioseismic observations revealed a subsurface rotational shear layer extending to a depth of 5\% of the solar surface (Schou et al. 1998).

Results from the semi-global direct numerical simulations (DNS) show that in addition to the dominant dynamo mode with a longer period, which is generated in the region where the equator-ward migration of the field is observed near the surface, there is a weaker pole-ward migrating dynamo mode with a shorter period working below the top of the domain (Käpylä et al. 2016). On the other hand, global magnetohydrodynamic (MHD) simulations (Strugarek et al. 2018) revealed that there are two types of cycles. These cycles are non-linearly coupled in the turbulent convective envelopes. The longer cycle originates from the bottom of the convection zone, while the shorter cycle is generated in the subsurface layers of the domain.

The solar dynamo is the physical mechanism, where the motion of the electrically conducting fluid, that is, the solar plasma, can support a self-excited dynamo against ohmic dissipation and maintains the global magnetic field in the convective envelope of the Sun (Parker 1955a,b). This dynamo generates and governs the spatiotemporal evolution of the magnetic activity of the Sun. A large-scale magnetic field can be generated via rotating, stratified, and electrically conducting turbulence. This process is generally referred to as the $\alpha$-effect and converts kinetic energy of the convection into magnetic energy. The precise nature of these non-dissipative turbulence effects and the $\alpha$-effect in particular, are still debated.

There are two basic processes involved in exciting an oscillatory self-sustaining dynamo: (i) generation of a toroidal field from a pre-existing poloidal field, and (ii) re-generation of the poloidal field from the generated toroidal field. The shearing of any poloidal field by solar differential rotation can generate a toroidal magnetic field. This process is known as the $\Omega$-effect. As for re-generating the poloidal field from the toroidal one, two of the most promising mechanisms can be described as (i) the effect of rotating stratified turbulence, where helical twisting of the toroidal field lines by the Coriolis force generates a poloidal field (turbulent $\alpha$-effect) (Parker 1955a,b; Brandenburg \& Subramanian 2005), and (ii) the Babcock-Leighton (BL) mechanism (Babcock 1961; Leighton 1964, 1969; Charbonneau 2014). In the BL mechanism, the poloidal field is generated upon the emergence of active regions by uplifting toroidal flux. Diffusion and advection lead to a net transport of flux of predominantly one polarity to the poles, if the active regions are tilted with regards to the east-west direction (Babcock 1961; Leighton 1964; Wang et al. 1989; Wang \& Sheeley 1991).

The inclusion of a poleward surface meridional flow along with an equator-ward deep-seated meridional flow led to the development of the so-called flux transport (FT) dynamo models (Wang et al. 1991; Dikpati \& Charbonneau 1999; Nandy \& Choudhuri 2001). The poleward meridional flow on the surface transports the poloidal field sources to the solar poles and causes the polarity reversal at sunspot maximum (Choudhuri et al. 1995; Dikpati \& Charbonneau 1999). The meridional flow then penetrates below the base of the convection zone and is responsible for the generation and equator-ward propagation of the bipolar activity structures at low latitudes at the solar surface (Dikpati \& Charbonneau 1999; Nandy \& Choudhuri 2001, 2002). There are several types of FT dynamo model, which produce the poloidal field either via a pure BL-mechanism or a pure $\alpha$-turbulent effect operating in the tachocline, or, alternatively, in the whole con-
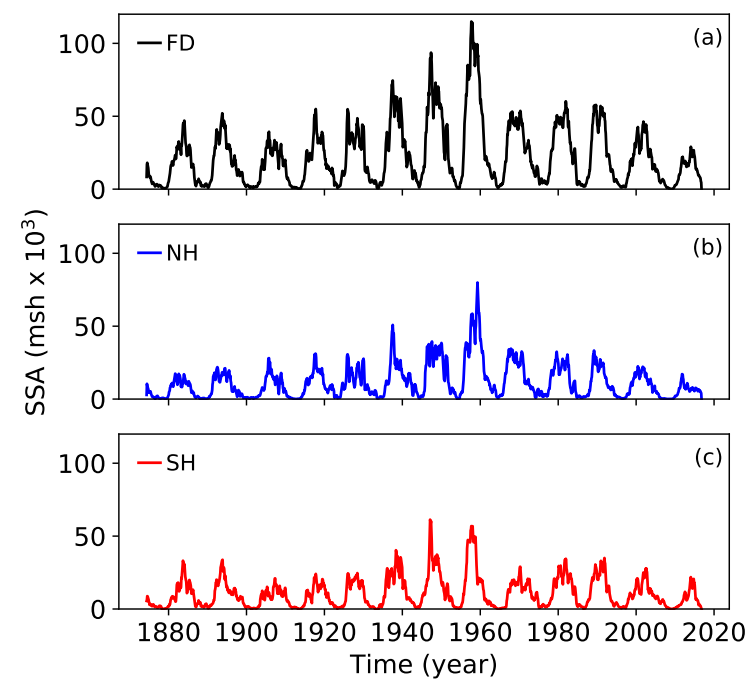

Fig. 1. Sunspot areas in millionths of solar hemisphere for the full disk $(a)$, and northern $(b)$ and southern $(c)$ solar hemispheres.

vection zone. More recently, dynamo models with $\alpha$-turbulence and BL-mechanisms operating simultaneously as poloidal field sources have also emerged (Dikpati \& Gilman 2001; Belucz \& Dikpati 2013; Passos et al. 2014).

In this study, we investigate the physical mechanisms that could lead to the observed QBOs using solar dynamos. We first analyse sunspot area (SSA) data to decipher the spatiotemporal features of the QBOs in the full disk as well as in the northern and southern hemispheres (Sect. 2). We then describe briefly the fully non-linear flux transport dynamos with two prevailing approaches to generating a poloidal field from an existing toroidal field: (i) the BL mechanism (Sect. 3.1) and (ii) the turbulent $\alpha$ mechanism (Sect. 3.2). Subsequently, we analyse the simulated data from the two dynamo models to study the possible underlying physics responsible for the spatiotemporal behaviour of the QBO and give the results in Sect. 4. We discuss the results and conclude in Sect. 5.

\section{The QBOs from sunspot areas}

To study the spatiotemporal behaviour of the QBO in solar activity, we used publicly available corrected daily sunspot area data $^{1}$ spanning 1874-2016. We then calculated the total sunspot areas in the full disk, the northern hemisphere, and the southern hemisphere. In addition, we smoothed the data using a moving average with a window length of 6 months to avoid high-frequency variations.

The multi-peak structure during the Schwabe-cycle maxima, which is thought to be related to the QBOs, can be observed in the total sunspot areas in the full disk, and in the northern and southern hemispheres (Fig. 1). Prior to further analyses, we standardised each SSA data set: full disk, northern and southern hemispheres according to their individual mean and standard deviation values.

To further investigate the behaviour of the QBO signal in the SSA data, we first calculated their Fourier power spectral densities (PSDs). To evaluate the significance of each of the peaks in the calculated Fourier PDSs, we calculated their theoretical Fourier red-noise PSDs based on the lag-1 auto-correlation coefficients of each SSA dataset. We then multiplied each

1 https://solarscience.msfc.nasa.gov/greenwch. shtml 


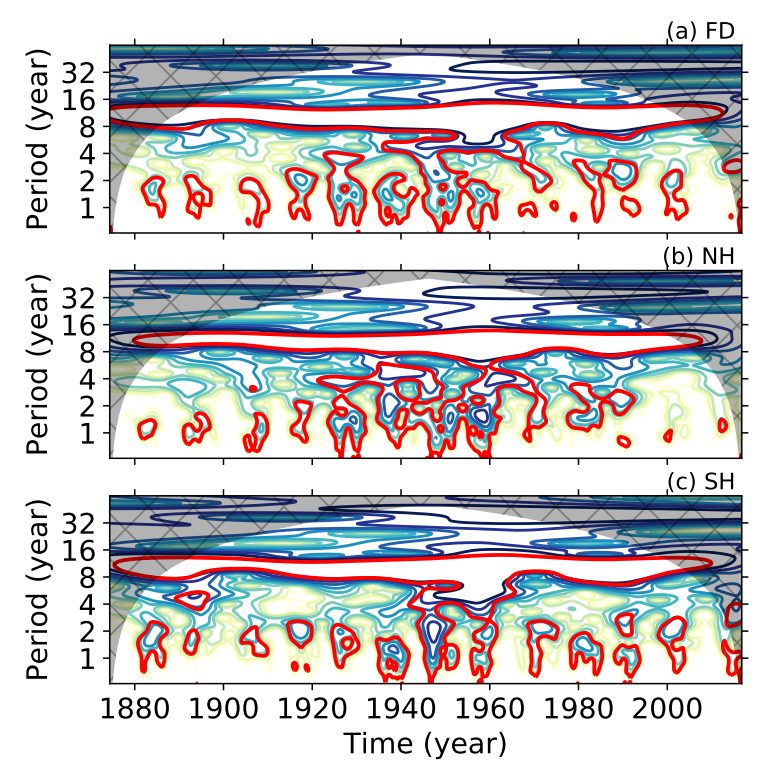

Fig. 2. Continuous wavelet spectra of the SSA data in the full disk (a), northern hemisphere $(b)$, and southern hemisphere $(c)$. Thick red contours indicate the significance level of 0.05 .

spectrum with the 95 th percentile value for $\chi^{2}$ distribution (Eqs. (16) and (17) in Torrence \& Compo 1998), indicating significance levels of 0.05 . The results show that the main period in the SSA data sets is $\sim 11$ years, corresponding to the Schwabe cycle. Additionally, there are shorter periodicities present in the SSA data below 5 years, which are not the harmonics of the main period of $\sim 11$ years and are significant at the 0.05 level. These shorter periods indicate the presence of the QBOs in hemispheric and full disk SSA data (Fig. A.1).

Additionally, we calculated the continuous wavelet spectra of each standardised SSA dataset using the method provided by Torrence \& Compo (1998) to investigate the temporal behaviour of the QBO signal. The temporal behaviour of the QBO signal in the wavelet spectrum of the full disk SSA data shows an inphase relationship with the Schwabe cycle, where the power of the QBO signal is more prominent during the Schwabe cycle maxima, and the QBO signal vanishes during the Schwabe cycle minima. Following 1960, the QBO signal during the Schwabe minima vanishes again, and after around 2000, the QBO signal is not present in the SSA data at all (Fig. 2a).

The QBO signal in the northern hemisphere, similar to the full disk, shows an in-phase relationship with the Schwabe cycle, where it is a continuous signal for the period extending from around 1920 to around 1960. It again becomes an intermittent signal in-phase with the Schwabe maxima after around 1960. Different from the full disk data however, the power of the QBO signal in the northern hemisphere becomes longer around 1990 before weakening around 2000; although weak, it comes back around 2015 (Fig. 2b). The QBO signal in the southern hemisphere, on the other hand, is intermittent and in-phase with the Schwabe cycle maxima throughout the study period. The QBO signal in the southern hemisphere around 2015 seems stronger compared with the signal in the northern hemisphere (Fig. 2c).

\section{The dynamo models}

To investigate the physical nature of the QBO signal observed across from the subsurface layers of the Sun to neutron counting rates measured on Earth, we use an FT dynamo model. This model uses the mean field differential rotation and meridional circulation model of Rempel (2005a), which is also coupled with the axisymmetric mean field induction equation (Rempel 2006). The $\Lambda$-mechanism in the model, which is responsible for the generation of the mean field differential rotation and meridional circulation is updated following Kitchatinov \& Rüdiger (2005). Although the large-scale flow field alone does not act as a dynamo, it does amplify and advect magnetic fields. It is modified, on the other hand, by the action of Lorentz forces exerted by the fields generated.

Updating the $\Lambda$-mechanism in the model using the angular momentum transport equations given in Kitchatinov \& Rüdiger (2005) led us to use a different set of parameters, which are given in Inceoglu et al. (2017). The computational domain for our dynamo models extends in latitude from the southern to the northern pole and in radius from $r=0.65 R_{\odot}$ to $0.985 R_{\odot}$. For a more detailed information on the dynamo model, we refer the reader to Rempel (2005a, 2006).

The computed reference differential rotation and meridional flow radial profiles and their contour plots are shown in Fig. 3. The differential rotation contours and profile, which are given in units of $\mathrm{nHz}$, show a subsurface shear-layer (Figs. 3a and c), which is in agreement with the helioseismic observations (Thompson et al. 2003; Howe 2009). There is one meridional circulation cell in each hemisphere (shown as $r \sin \theta P S I$, where $P S I$ is the stream function, in Fig. $3 \mathrm{~b}$ ) and the circulation speed on top of the domain at $45^{\circ} \mathrm{N}$ latitude is $\sim 16 \mathrm{~m} \mathrm{~s}^{-1}$ (Fig. 3d).

\subsection{Babcock-Leighton dynamo models}

Dynamo action is accomplished by adding a source term $S\left(r, \theta, B_{\phi}\right)$, mimicking the BL-effect in the induction equation (see Appendix B and Rempel 2006, for details), which is given as:

$S\left(r, \theta, B_{\phi}\right)=\alpha_{0} \bar{B}_{\phi, b c}(\theta) f_{\alpha}(r) g_{\alpha}(\theta)$,

with

$f_{\alpha}(r)=\max \left[0,1-\frac{\left(r-r_{\max }\right)^{2}}{d_{\alpha}^{2}}\right]$

$g_{\alpha}(r)=\frac{(\sin \theta)^{4} \cos \theta}{\max \left[(\sin \theta)^{4} \cos \theta\right]}$

$\bar{B}_{\phi, \mathrm{bc}}(\theta)=\int_{r_{\min }}^{r_{\max }} \mathrm{d} r h(r) B_{\phi}(r, \theta)$

where $d_{\alpha}=0.05 R_{\odot}$. This confines the poloidal source term above $r=0.935 R_{\odot}$, where it peaks at $r_{\max }$. The function $h(r)$ is an averaging kernel with $\int_{r_{\min }}^{r_{\max }} \mathrm{d} r h(r)=1$. The boundary condition for the model is that the vector potential $A$ and the toroidal field $B_{\phi}$ obey $A=B_{\phi}=0$ and $\partial A / \partial \theta=B_{\phi}=0$ at the poles and equator, respectively (see Appendix B and Rempel 2006, for details).

The BL $\alpha$-effect in our simulations is non-local, meaning that it operates on the surface (Fig. 4a) and is proportional to the toroidal field strength at the base of the convection zone averaged over the interval $\left[0.71 R_{\odot}, 0.76 R_{\odot}\right]$.

The amplitude of $0.4 \mathrm{~ms}^{-1}$ for the $\alpha$-effect coefficient, $\alpha_{0}$, provided stable anti-symmetric oscillatory behaviour as observed on the Sun. The butterfly diagram computed based on the given configuration at $0.71 R_{\odot}$ shows that the solar cycle starts at around $50^{\circ}$ latitude at each hemisphere and the magnetic activity propagates equator-ward and poleward (Fig. 5a) 

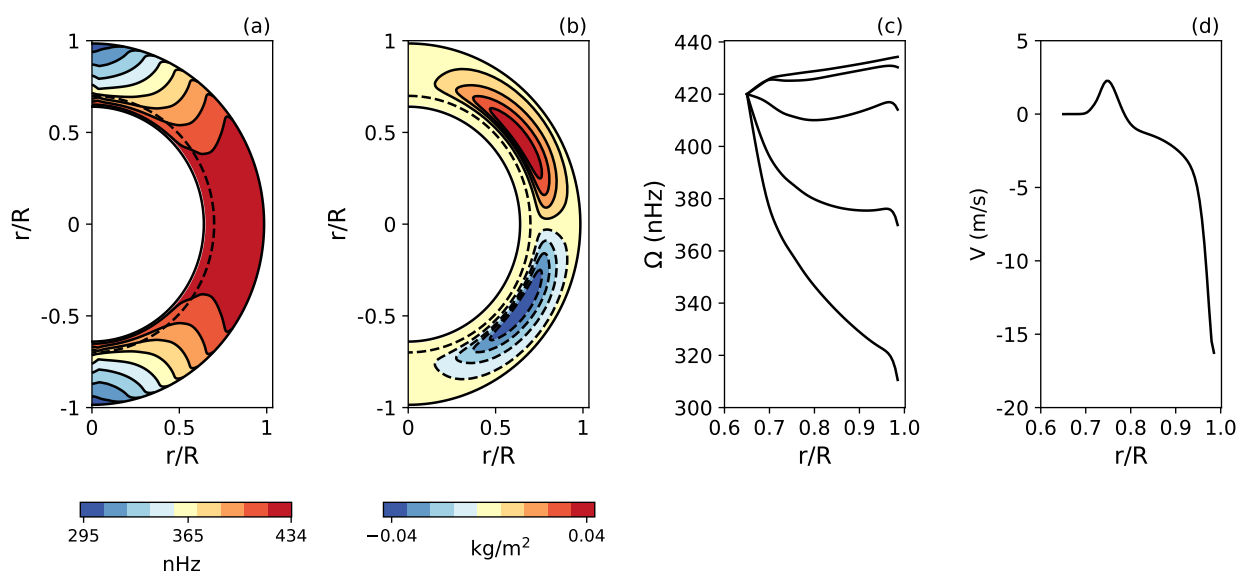

Fig. 3. Reference differential rotation (a) and meridional flow in $r \sin \theta P S I$, where $P S I$ is the stream function $(b)$ contour plots. We note that the meridional flow contours are normalised according to the velocity unit $\left(\left(p_{\mathrm{bc}} / \varrho_{\mathrm{bc}}\right)^{1 / 2}\right)$. We also show the radial profiles of the reference differential rotation and meridional circulation for the northern solar hemisphere, respectively $(c, d)$. The radial profile of the differential rotation $(c)$ is given at $0^{\circ}, 20^{\circ}, 40^{\circ}, 60^{\circ}$, and $80^{\circ}$ northern latitudes, from top to bottom, while the meridional circulation $(d)$ is given at $45^{\circ} \mathrm{N}$ latitude.

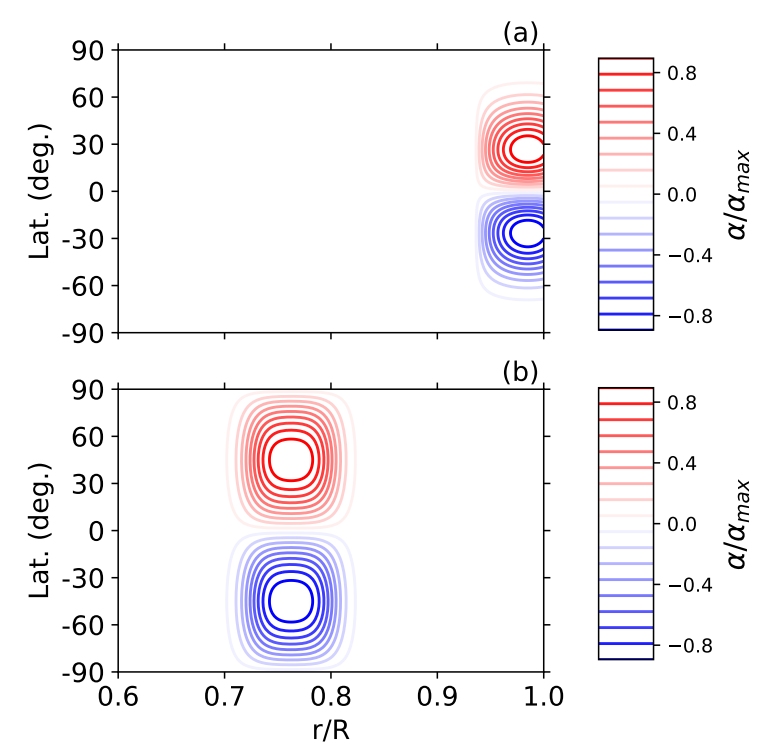

Fig. 4. Alpha effect contours for (a) the BL dynamo (Eqs. (2) and (3)) and (b) turbulent $\alpha$-dynamo (Eqs. (5) and (6)) models.

as the cycle progresses, resembling the observed sunspot cycles. The average strength of the generated magnetic field in the reference model is around $\left|B_{\phi}\right|=3.25$ Tesla, fluctuating between 0.90 and 5.34 Tesla.

\subsection{Turbulent $\alpha$-dynamo models}

The turbulent $\alpha$-effect for the dynamo to generate a poloidal field from a pre-existing toroidal field is taken following Dikpati \& Gilman (2001) (also see Appendix B for details):

$f_{\alpha}(r)=\frac{1}{4}\left[1+\operatorname{erf}\left(\frac{r-r_{2}}{d_{2}}\right)\right]\left[1-\operatorname{erf}\left(\frac{r-r_{3}}{d_{3}}\right)\right]$,

with

$g_{\alpha}(r)=\frac{\sin \theta \cos \theta}{\max [\sin \theta \cos \theta]}$,

where $r_{2}=0.725 R_{\odot}, r_{3}=0.80 R_{\odot}$, and $d_{2}=d_{3}=0.02 R_{\odot}$. This function constrains the turbulent $\alpha$-effect to a thin layer at

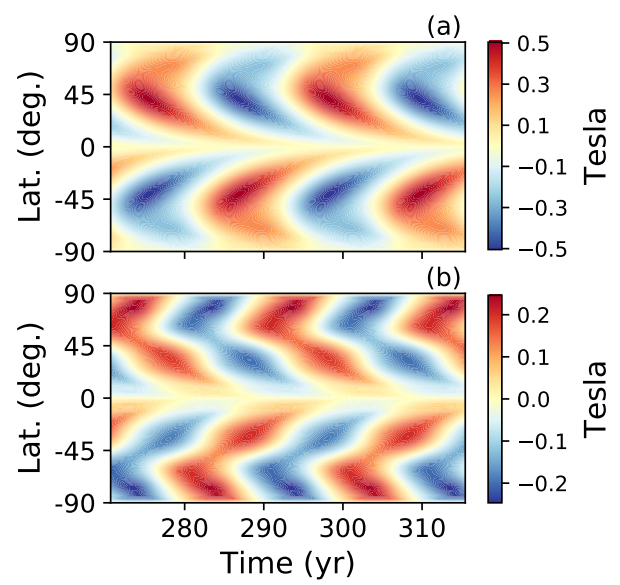

Fig. 5. The reference butterfly diagram at $0.71 R_{\odot}$ obtained from $(a)$ the BL dynamo and $(b)$ turbulent $\alpha$-dynamo models.

the base of the convection zone, just above the tachocline, and to mid-latitudes (Fig. 4b).

We chose the amplitude of the turbulent $\alpha$-effect coefficient as $\alpha_{0}=0.04 \mathrm{~ms}^{-1}$, which provided stable anti-symmetric oscillatory solutions. The butterfly diagram calculated based on the given parameters at $0.71 R_{\odot}$ shows that the solar cycles start around $60^{\circ}$ latitude at each hemisphere and the magnetic activity propagates towards the poles and the equator as the cycles progress (Fig. 5b). The average strength of the generated magnetic field in the reference turbulent $\alpha$-model is around $\left|B_{\phi}\right|=$ 0.96 Tesla, fluctuating between 0.65 and 1.16 Tesla. As opposed to the BL-dynamo, the turbulent $\alpha$-dynamo exhibits stronger polar branches, whereas equator-ward branches are stronger in the BL-dynamo (Figs. 5a and b).

\section{Analyses and Results}

\subsection{Babcock-Leighton dynamos}

We used BL dynamos to investigate the physical mechanisms that could lead to the observed relationships in the SSA data sets. To obtain the QBO signals in the BL dynamos, we gradually increased the amplitude of the BL $\alpha$-effect coefficient from $0.4 \mathrm{~ms}^{-1}$, which provides stable oscillatory solutions (Fig. 5a), 


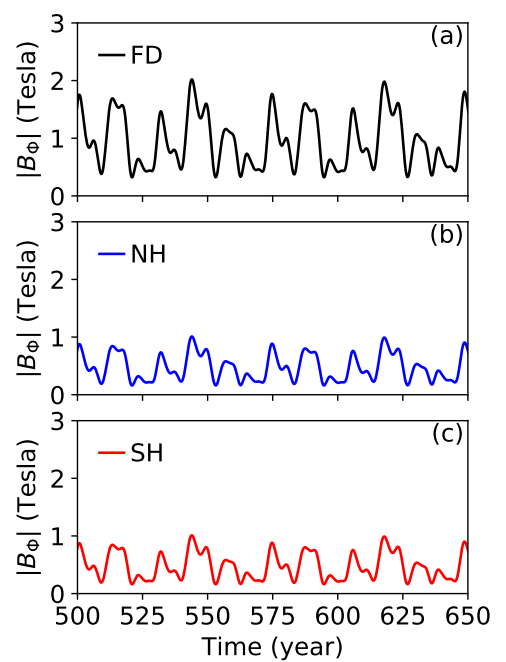

Fig. 6. Magnetic field strengths from the BL dynamo for $(a)$ the full disk (black), and for (b) the northern (blue) and (c) southern (red) solar hemispheres.

to $1.4 \mathrm{~ms}^{-1}$ with $0.1 \mathrm{~ms}^{-1}$ increments. Dynamo simulations were not achievable using any larger amplitudes of the BL $\alpha$-effect because of numerical resolution limitations.

We then calculated the total magnetic field strengths at $0.71 R_{\odot}$, which we used for the further analyses. The amplitudes of the total magnetic field strength for the full disk in simulations decreased from above $\left|B_{\phi}\right|=5$ Tesla to around $\left|B_{\phi}\right|=2$ Tesla as we increased the amplitude of the BL $\alpha$-effect coefficient from $0.4 \mathrm{~ms}^{-1}$ to $1.4 \mathrm{~ms}^{-1}$. This is a direct result of the Lorentz force feedback acting as the saturation mechanism. Here we present the results from the BL dynamo with the $\alpha$-effect coefficient amplitude of $1.4 \mathrm{~ms}^{-1}$.

We calculated the total magnetic field strengths for the northern and southern hemispheres (Fig. 6) at $0.71 R_{\odot}$. The $\mathrm{BL}$ dynamo shows similar behaviour between the magnetic field strengths of the northern and southern hemispheres on the contrary to the hemispheric SSA data.

To investigate whether the BL-dynamo simulation shows a QBO-like behaviour, we calculated the Fourier power spectral densities using the standardised values of the BL-dynamo data for the full disk, and northern and southern hemispheres. Similarly to the SSA data, we standardised the data using their individual mean and standard deviation values prior to the Fourier analysis. The results from the Fourier analyses for each data set show that the period with the highest power spectral densities are about $\sim 14.8$ years for the full disk and the northern and southern hemispheres. There are also signs of periods below 5 years in the Fourier power spectral densities that can be associated with the QBO. These periods are close to the 0.05 significance limit for the full disk, and northern and southern hemispheres (Fig. A.2).

Following the Fourier analyses, we calculated the continuous wavelet spectra of the simulated data set (Fig. 7). The results from the wavelet analyses show that there are no periods below about 4 years in the data sets. However, there are intermittent signals of a shorter period (SP) between 4 and 8 years in addition to the more continuous longer period (LP) of around 15 years in full disk and in both hemispheres (Fig. 7a and b).

To investigate whether the non-linear interplay between the magnetic and the flow fields might be the reason for the generation of shorter periods observed in the full disk and the hemispheric continuous wavelet spectra (Figs. 7a and b), we analysed meridional circulation and differential rotation values.
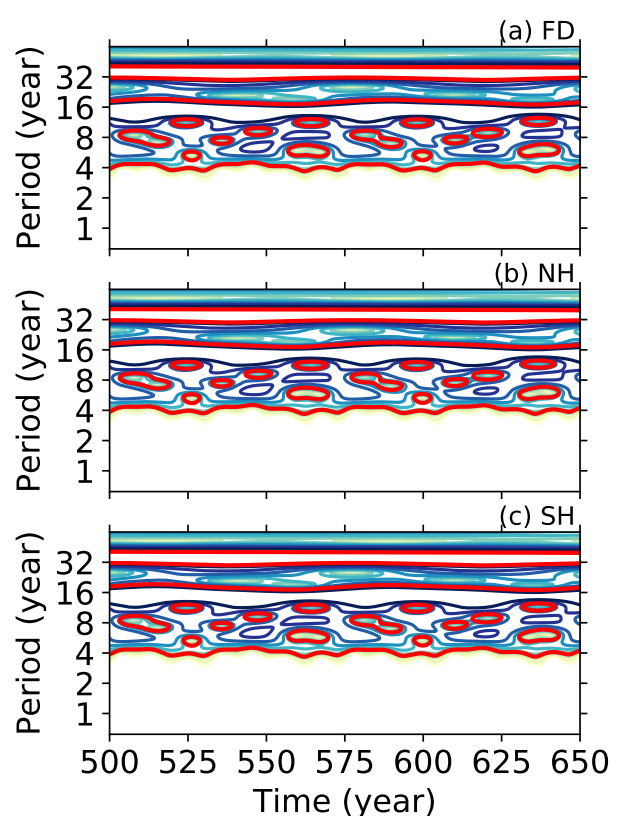

Fig. 7. Continuous wavelet spectra of the $\left|B_{\phi}\right|$ data from BL-dynamo in the full disk $(a)$, and northern $(b)$, and southern $(c)$ hemisphere. Thick red contours indicate the significance level of 0.05 .
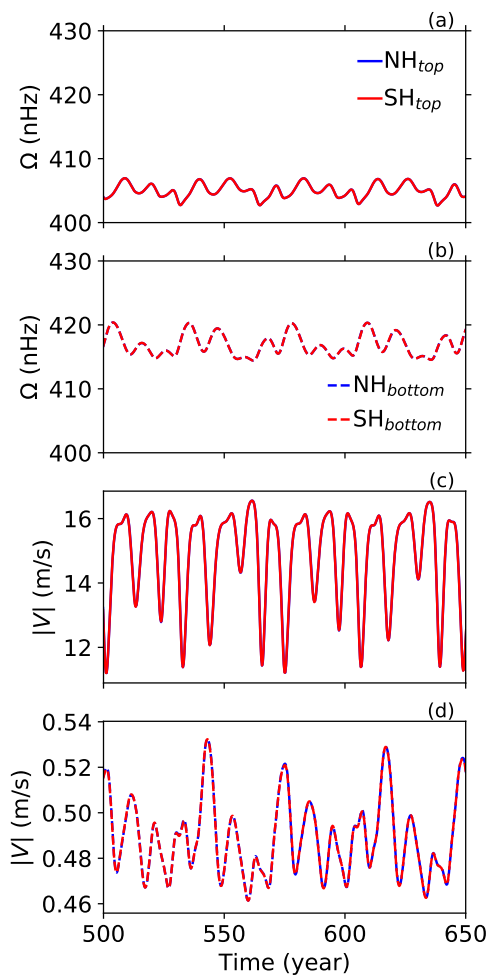

Fig. 8. Differential rotation $(a, b)$ and meridional circulation $(c, d)$ rates at the top of the domain $0.985 R_{\odot}$ (lines) and at the bottom of the convection zone $0.71 R_{\odot}$ (dashed lines) at $45^{\circ}$ latitude $\mathrm{N}$ (blue) and $\mathrm{S}$ (red) for the BL-dynamo.

These values are obtained at $45^{\circ}$ latitude $\mathrm{N}$ and $\mathrm{S}$ and at $0.985 R_{\odot}$ and $0.71 R_{\odot}$ from our BL-dynamo (Fig. 8). The differential rotation values at the top of the domain and at the bottom of the convection zone for both hemispheres are the same and exhibit small amplitude variations (Figs. 8a and b). The meridional circulation values on top of the domain show variations with 


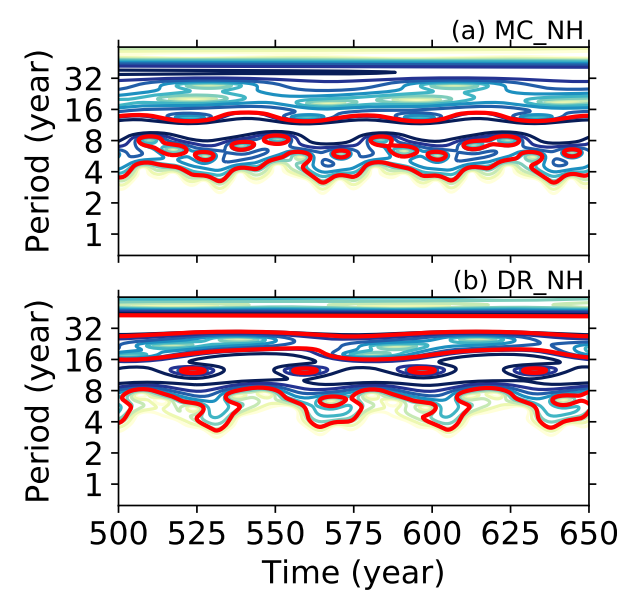

Fig. 9. Continuous wavelet spectra of the meridional circulation (a) and differential rotation $(b)$ values calculated at $45^{\circ}$ latitude $\mathrm{N}$ and at $0.985 R_{\odot}$ from the BL-dynamo. Thick red contours indicate the significance level of 0.05 .

maximum amplitude of $4 \mathrm{~m} \mathrm{~s}^{-1}$, whereas the maximum amplitude change for the bottom of the convection zone is around $0.04 \mathrm{~m} \mathrm{~s}^{-1}$ (Figs. 8c and d).

We then calculated the wavelet spectra of the differential rotation and meridional circulation on top of the domain, as the meridional circulation shows very small amplitude variations at the bottom of the convection zone. Prior to the wavelet analyses, we first standardised the meridional circulation and differential rotation values. We then calculated their wavelet spectra. The meridional circulation in both hemispheres exhibits intermittent longer-term variations above 8 years and a shorter-term variation of around 4 years, the latter being absent from the differential rotation rates (Fig. 9).

\subsection{Turbulent $\alpha$-dynamos}

In addition to the BL-dynamos, we also generated the magnetic field strengths using the turbulent $\alpha$-dynamos to study the physical mechanisms leading to the spatiotemporal relationships observed in the SSA data. To produce the observed features in the SSA data, similar to the BL-dynamos, we gradually increased the amplitude of the turbulent $\alpha$-effect coefficient from $0.04 \mathrm{~ms}^{-1}$, which gives the stable oscillatory solutions (Fig. 5b), to $0.2 \mathrm{~ms}^{-1}$ with $0.01 \mathrm{~ms}^{-1}$ increments. Dynamo simulations were not feasible using any larger amplitudes of the turbulent $\alpha$-effect due to numerical resolution limitations.

The amplitudes of the total magnetic field strength calculated for the full disk in simulations have increased from below $\left|B_{\phi}\right|=1.2$ Tesla to below around $\left|B_{\phi}\right|=8$ Tesla as the amplitude of the turbulent $\alpha$-effect coefficient increased from $0.04 \mathrm{~ms}^{-1}$ to $0.2 \mathrm{~ms}^{-1}$. Throughout the different dynamos with increasing turbulent $\alpha$-effect coefficient amplitudes, the corresponding simulated butterfly diagrams showed different symmetries, from total symmetry to mixed-parity, where the anti-symmetry around the equator prevails. Here we present the results from the turbulent $\alpha$-dynamo with the $\alpha$-effect coefficient amplitude of $0.2 \mathrm{~ms}^{-1}$ and mixed parity with prevailing anti-symmetry.

We calculated the total magnetic field strengths for the northern and southern hemispheres at $0.71 R_{\odot}$ (Fig. 10). Different from the SSA data but similar to the BL-dynamo, the magnetic field strengths calculated for the northern and southern hemispheres show a decoupled behaviour, which becomes prominent for the period between around 1650 and 1710 .

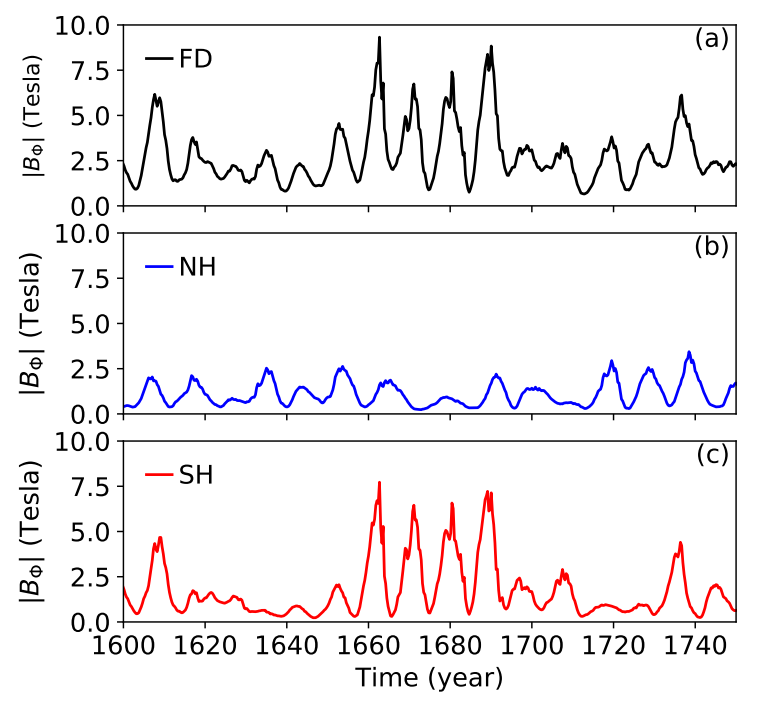

Fig. 10. Magnetic field strengths from turbulent $\alpha$-dynamo for $(a)$ the full disk (black), and the $(b)$ northern (blue) and (c) southern (red) solar hemispheres.

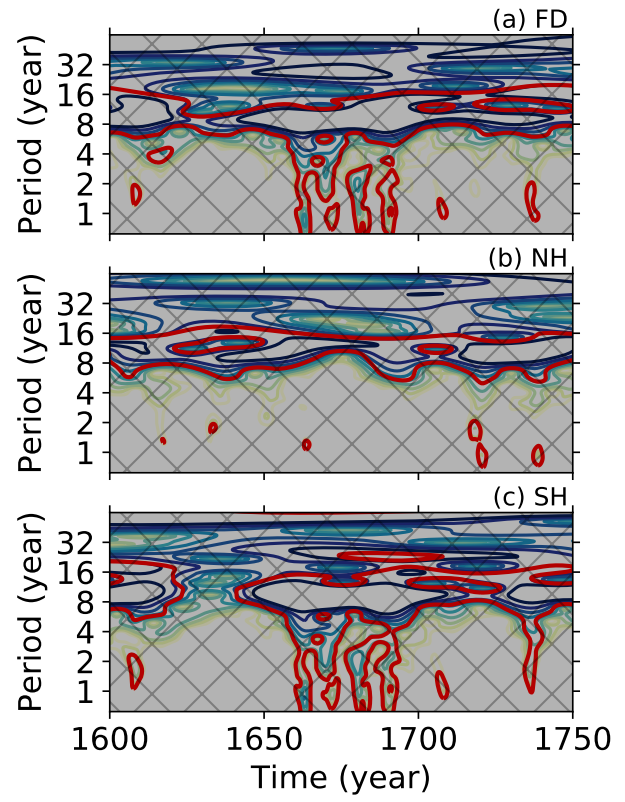

Fig. 11. Continuous wavelet spectra of the $\left|B_{\phi}\right|$ data from turbulent $\alpha$ dynamo in the full disk $(a)$, and the $(b)$ northern and southern $(c)$ hemispheres. Thick red contours indicate the significance level of 0.05 .

To investigate whether the turbulent $\alpha$-dynamo shows a QBO-like behaviour, we calculated the Fourier power spectral densities using the standardised values of the total magnetic field calculated for the full disk and for the northern and southern hemispheres. Similar to the SSA data, we standardised the simulated data using their individual mean and standard deviation values prior to the Fourier analysis. The results from the Fourier analyses show that the period with the highest power spectral densities are about $\sim 9.5$ years across all data together with periods below 5 years that can be associated with the QBO in the Fourier power spectral densities. These periods are statistically significant at the 0.05 level with changing amplitudes across the full disk, and the northern and southern hemispheres (Fig. A.3).

In addition to the Fourier analyses, we calculated the continuous wavelet spectra of the simulated data set. The results of the wavelet power spectra show that there are periods around 

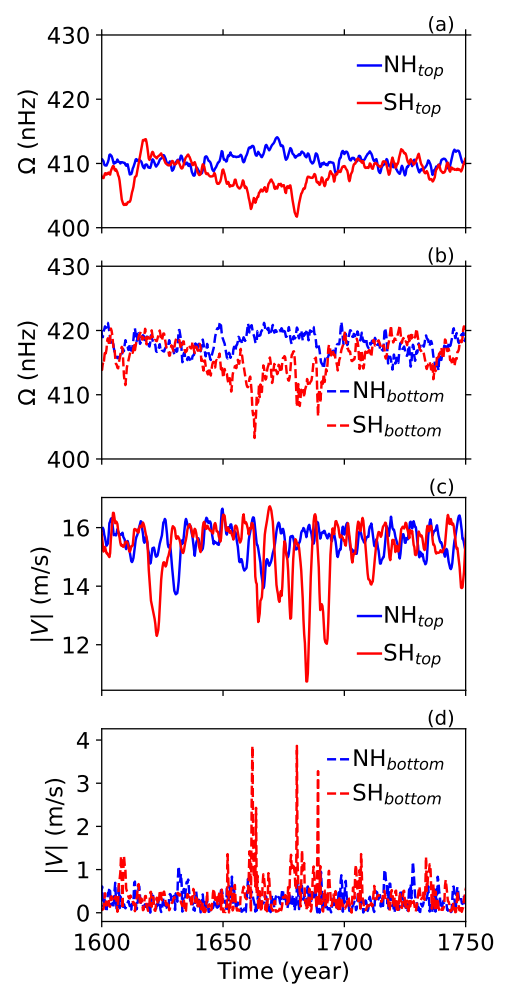

Fig. 12. Differential rotation $(a, b)$ and meridional circulation $(c, d)$ rates at the top of the domain $0.985 R_{\odot}$ (lines) and at the bottom of the convection zone $0.71 R_{\odot}$ (dashed lines) at $45^{\circ}$ latitude $\mathrm{N}$ (blue) and $\mathrm{S}$ (red) for the turbulent $\alpha$-dynamo.

9.5 years across all data sets as well as periods below 5 years, which can be associated with the QBO signal in the SSA data (Fig. 11). These shorter periods as well as the longer periods are intermittent throughout the simulation period and exhibit an in-phase variation. The presence of the shorter period is less pronounced in the northern hemisphere than in the southern hemisphere. The intermittency of both the shorter and longer periods appears to originate from variations in the total magnetic field strength calculated for the full disk, and for the northern and southern hemispheres separately when compared with Fig. 10.

To investigate the non-linear interaction between the magnetic field and flow fields as an underlying mechanism to the observed QBO-like signal in our turbulent $\alpha$-dynamo (Fig. 11), we study the variations in the meridional circulation and differential rotation values at $45^{\circ}$ latitude $\mathrm{N}$ and $\mathrm{S}$ and on top of the domain at $0.985 R_{\odot}$, and at the bottom of the convection zone at $0.71 R_{\odot}$ (Fig. 12). The differential rotation rates in the southern hemisphere, both on top of the domain and at the bottom of the convection zone, display an anti-correlated relationship with those in the northern hemisphere (Figs. 12a and b). The differential rotation rates show their maximum variation of around $15 \mathrm{nHz}$ between the dates 1650 and 1700 at the bottom of the convection zone, while the amplitude of variation in differential rotation rates in the northern hemisphere is around $5 \mathrm{nHz}$. Similar to the differential rotation values, the meridional circulation speed in the southern hemisphere shows larger variations compared with that in the northern hemisphere. The meridional circulation on top of the domain slows down by $4 \mathrm{~m} \mathrm{~s}^{-1}$ in the southern hemisphere, while it experiences episodes with increased speed by more than $3 \mathrm{~m} \mathrm{~s}^{-1}$ at the bottom of the convection zone (Figs. 12c and d) between 1650 and 1700. Interestingly, this period also shows stronger magnetic field strengths

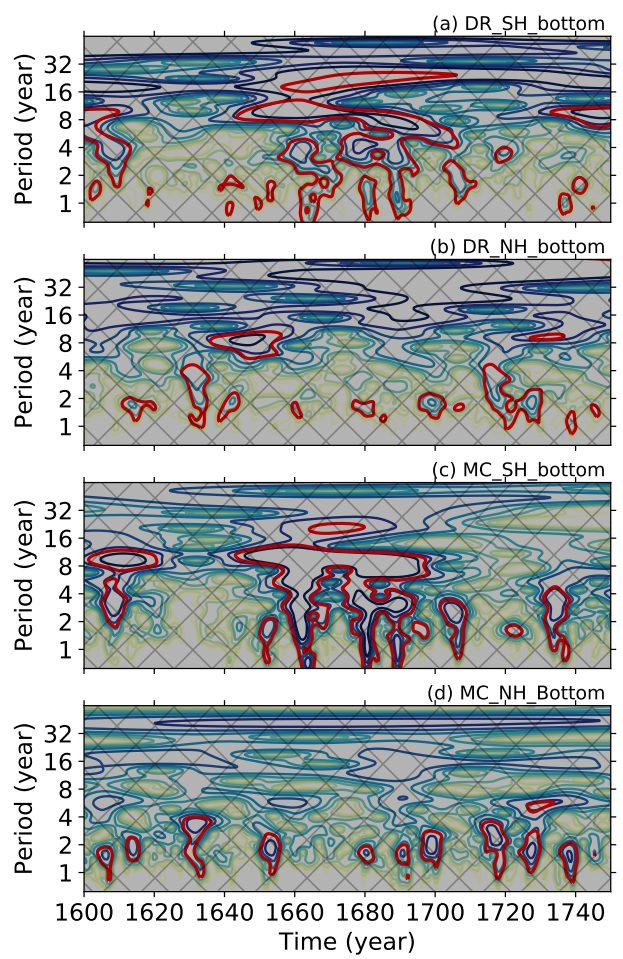

Fig. 13. Continuous wavelet spectra of the meridional circulation $(a, b)$ and differential rotation $(c, d)$ values calculated at $45^{\circ}$ latitude $\mathrm{N}$ and $\mathrm{S}$ and at $0.71 R_{\odot}$ from the turbulent $\alpha$-dynamo. Thick red contours indicate the significance level of 0.05 .

together with QBO-like oscillations only in the southern hemisphere, whereas the northern hemisphere mainly shows oscillations with a period of around 10 years (Figs. $11 \mathrm{~b}$ and c).

To investigate whether the meridional circulation and differential rotation show any QBO-like signal, we calculated their wavelet power spectra. Prior to the wavelet analyses, we first standardised the meridional circulation and differential rotation values using their individual mean and standard deviation values. The results for the bottom of the convection zone show that both the meridional circulation and the differential rotation show intermittent periods of between 1 and 4 years at the $p<0.05$ level (Fig. 13). In the wavelet spectrum of the meridional circulation values of the southern hemisphere, statistically significant and powerful periods extending from around 9 years to below 2 years can be observed in approximately the year 1650 (Fig. 13b). Similarly, these periods can also be observed in the magnetic field strengths in the full disk and the southern hemisphere (Figs. 11a and c). The intermittency in the QBO-like signals from both the magnetic field and flow fields seems to vary simultaneously (Figs. 11 and 13).

\section{Discussion and conclusions}

The results from the Fourier and wavelet analyses for the sunspot areas in the full disk, and northern and southern hemispheres reveal that the QBOs are present in sunspot area data with slight differences in temporal and hemispheric behaviours. The observed QBOs generally show an intermittent behaviour with maximum power occurring during the Schwabe cycle maxima, while the QBOs vanish during the Schwabe cycle minima. The QBO signals in the northern hemisphere become considerably less prominent than those observed in the southern hemisphere for the period after around 1990. This feature points to a 
decoupled behaviour of the solar hemispheres, which was also observed for the magnetic energies (Inceoglu et al. 2017). The intermittent behaviour of the QBO was also observed in other solar indices, such as the dipole magnetic moment and open magnetic flux of the Sun (Wang \& Sheeley 2003), global solar magnetic field (Ulrich \& Tran 2013), and in the frequencies of the acoustic oscillations (Broomhall et al. 2012; Simoniello et al. 2012, 2013, 2016).

We therefore decided to use these observational findings to investigate the physical mechanisms responsible for the generation and temporal and hemispheric evolution of the QBOs. To achieve this, we used fully non-linear flux transport dynamo models with two prevailing approaches to generating a poloidal field from an existing toroidal field: a non-local BL model and a turbulent $\alpha$-dynamo model.

The results from the non-local BL-dynamo runs show that it generally fails to produce QBO-like shorter cycles and the slightly decoupled behaviour of the solar hemispheres. We also investigated whether the dynamo runs with smaller $\alpha$-effect amplitudes show any QBO-like oscillations. As we increased the BL-effect amplitude from $\alpha_{0}=0.4 \mathrm{~ms}^{-1}$ to $1.4 \mathrm{~ms}^{-1}$ at increments of $0.1 \mathrm{~ms}^{-1}$, the main oscillation that has a period of 11.4 years becomes more perturbed where it shows very small amplitude variations superimposed on the main oscillation. However, none of these runs showed QBO-like oscillations.

The turbulent $\alpha$-dynamo on the other hand generated QBOlike intermittent oscillations together with the Schwabe-like cycles as well as the decoupled behaviour of the solar hemispheres. To ensure the QBO-like fluctuations do not result from numerical noise, we also re-ran the dynamo code with doubled spatial and temporal resolution. The results from the highresolution run showed that the QBO-like oscillations are indeed a result of the non-linear interplay between the magnetic field and flow fields. The results from the turbulent $\alpha$-dynamo also show that the meridional flow is more strongly impacted by short-term field variability than the differential rotation values (visible around 1700 in Fig. 13). This is expected since the meridional flow, which is a strongly driven weak flow, has much less kinetic energy and a much shorter response timescale (Rempel 2005b). Similar to the BL-dynamos, we also investigated the effect of the amplitude of turbulent $\alpha$-effect coefficient, ranging between $0.04 \mathrm{~ms}^{-1}$ and $0.2 \mathrm{~ms}^{-1}$. For the weakest coefficient, the magnetic field strength oscillates with a period of 10 years without any perturbations. However, as we increase the amplitude of the turbulent $\alpha$-effect coefficient, the stable oscillation obtained for the amplitude of the turbulent $\alpha$-effect coefficient of $0.04 \mathrm{~ms}^{-1}$ started to exhibit variations that are superimposed and the amplitudes of these variations increased with the increasing alpha coefficient. After the alpha coefficient of $0.15 \mathrm{~ms}^{-1}$, the wavelet analyses started to show QBO-like oscillations.

The results from the BL- and turbulent $\alpha$-dynamos suggest an acute sensitivity to the detailed structure of the dynamo magnetic field. The shapes of our $\alpha$-effects both for the turbulent and BL $\alpha$-mechanisms, show localisation in latitude and radius to a specific degree, to ensure that the simulations show solar-like oscillations with solar-like butterfly diagrams. We tried several shapes for our $\alpha$-effects, especially for the turbulent $\alpha$, and concluded that the $\alpha$-effect shapes used in our simulations provided the best solar-like solutions. Such shapes are generally not supported by the 3D turbulent numerical simulations (Augustson et al. 2015; Simard et al. 2016; Warnecke et al. 2018).

Our results demonstrated that the non-local BL dynamo does not reproduce QBO-like features, while the turbulent $\alpha$-effect at the bottom of the convection zone is more effective in generating QBO-like oscillations. A comparison among the meridional circulation speeds, differential rotation rates, and the magnetic field strengths from the BL (Figs. 6 and 8) and the turbulent $\alpha$-dynamos (Figs. 10 and 12) revealed the importance of the differential rotation and meridional circulation rates at the bottom of the convection zone. This region is also very close to where our turbulent $\alpha$-dynamo operates, whereas the non-local BL-dynamo $\alpha$-effect operates on the surface. This might indicate that in the presence of the local turbulent $\alpha$-effect, the induced magnetic field will immediately lead to Lorentz force feedback on the differential rotation and meridional circulation on the bottom of the convection zone. In the case of the non-local BL $\alpha$-effect, which operates on the surface, the induced magnetic field has to be transported to the bottom of the convection zone by the meridional circulation and diffusion and then lead to Lorentz force feedback. This delayed response might be the reason for the absence of QBO-like oscillations in non-local BL dynamos.

Additionally, we investigated the effects of the statistical significance levels of the results from the Fourier and wavelet analyses; we ran them again with significance levels of 0.10 and 0.01 . These runs showed very similar results, with only slightly different significance contours, which indicate that our results are robust.

The results from the acoustic-mode frequency shifts suggest that the QBOs might be driven in the near surface layers where the ratio of the gas pressure to magnetic pressure, $\beta=$ $\left(P_{\text {gas }} / P_{\text {mag }}\right)$, is much smaller (Simoniello et al. 2013). The helioseismic results have been interpreted either as the presence of a secondary dynamo near the subsurface layers (Benevolenskaya 1998; Fletcher et al. 2010) or as the result of a beating between different magnetic configurations (Berdyugina \& Usoskin 2003; Simoniello et al. 2013). Further, semi-global DNS and global MHD simulations seem to generate shorter QBO-like cycles in the near-surface layers (Käpylä et al. 2016; Strugarek et al. 2018). Results from global MHD simulations also suggest that these two cycles are not necessarily generated by a single dynamo mechanism (Strugarek et al. 2018), indicating the possibility of a secondary dynamo working in the near surface layer of the Sun. In this region the ratio of the gas pressure to magnetic pressure dramatically decreases, indicating a change in the magnetic field strength or local topology (Simoniello et al. 2013). The near surface layer of the Sun also accommodates a rotational shear-layer (Schou et al. 1998).

In conclusion, our simulations show that the turbulent $\alpha$-dynamos with the Lorentz force seem more efficient in generating the observed temporal and spatial behaviour of the QBO signal compared with the non-local BL-dynamos. It could also be interesting to investigate whether equator-ward migration from the delay of flux rise to the surface rather than from the deep meridional flow (Fournier et al. 2018) could lead to stronger QBOs in BL-dynamos. However, to fully understand the specific physical mechanism leading to the presence of QBO-like signals in the turbulent $\alpha$-dynamos, and their absence in the non-local BL-dynamos, would require very detailed analyses of the terms in the dynamo equations, such as Lorentz force, shear, advection, and stretching. Additionally, to assess whether a secondary dynamo operating on the surface or in the near-surface layers could generate the observed features in the sunspot areas, a detailed study is necessary; for this, we plan to use dynamos with a turbulent $\alpha$-mechanism at the bottom of the convection zone and the BL-mechanism on the surface, as well as two turbulent $\alpha$-dynamos placed at the bottom and top of the convection zone. 
Acknowledgements. We thank Mausumi Dikpati for her useful comments We also thank the anonymous reviewer for the comments, which improved the manuscript. Funding for the Stellar Astrophysics Centre is provided by the Danish National Research Foundation (grant agreement no. DNRF106)

The National Center for Atmospheric Research is sponsored by the National Science Foundation.

\section{References}

Augustson, K., Brun, A. S., Miesch, M., \& Toomre, J. 2015, ApJ, 809, 149 Babcock, H. W. 1961, ApJ, 133, 572

Bazilevskaya, G., Broomhall, A.-M., Elsworth, Y., \& Nakariakov, V. M. 2014, Space Sci. Rev., 186, 359

Belucz, B., \& Dikpati, M. 2013, ApJ, 779, 4

Benevolenskaya, E. E. 1998, ApJ, 509, L49

Berdyugina, S. V., \& Usoskin, I. G. 2003, A\&A, 405, 1121

Brandenburg, A., \& Subramanian, K. 2005, Phys. Rep., 417, 1

Broomhall, A.-M., Chaplin, W. J., Elsworth, Y., \& Simoniello, R. 2012 MNRAS, 420, 1405

Charbonneau, P. 2014, ARA\&A, 52, 251

Choudhuri, A. R., Schussler, M., \& Dikpati, M. 1995, A\&A, 303, L29

Dikpati, M., \& Charbonneau, P. 1999, ApJ, 518, 508

Dikpati, M., \& Gilman, P. A. 2001, ApJ, 559, 428

Dikpati, M., McIntosh, S. W., Bothun, G., et al. 2018, ApJ, 853, 144

Fletcher, S. T., Broomhall, A.-M., Salabert, D., et al. 2010, ApJ, 718, L19

Fournier, Y., Arlt, R., \& Elstner, D. 2018, A\&A, 620, A135

Gleissberg, W. 1939, The Observatory, 62, 158

Howe, R. 2009, Liv. Rev. Sol. Phys., 6, 1

Inceoglu, F., Arlt, R., \& Rempel, M. 2017, ApJ, 848, 93

Inceoglu, F., Simoniello, R., Knudsen, M. F., \& Karoff, C. 2017, A\&A, 601, A51

Käpylä, M. J., Käpylä, P. J., Olspert, N., et al. 2016, A\&A, 589, A56

Kitchatinov, L. L., \& Rüdiger, G. 2005, Astron. Nachr., 326, 379

Kudela, K., Mavromichalaki, H., Papaioannou, A., \& Gerontidou, M. 2010, Sol. Phys., 266, 173
Leighton, R. B. 1964, ApJ, 140, 1547

Leighton, R. B. 1969, ApJ, 156, 1

Nandy, D., \& Choudhuri, A. R. 2001, ApJ, 551, 576

Nandy, D., \& Choudhuri, A. R. 2002, Science, 296, 1671

Pap, J., Tobiska, W. K., \& Bouwer, S. D. 1990, Sol. Phys., 129, 165

Parker, E. N. 1955a, ApJ, 122, 293

Parker, E. N. 1955b, ApJ, 121, 491

Passos, D., Nandy, D., Hazra, S., \& Lopes, I. 2014, A\&A, 563, A18

Rempel, M. 2005a, ApJ, 622, 1320

Rempel, M. 2005b, ApJ, 631, 1286

Rempel, M. 2006, ApJ, 647, 662

Schou, J., Antia, H. M., Basu, S., et al. 1998, ApJ, 505, 390

Schwabe, H. 1844, Astron. Nachr., 21, 233

Simard, C., Charbonneau, P., \& Dubé, C. 2016, Adv. Space Res., 58, 1522

Simoniello, R., Finsterle, W., Salabert, D., et al. 2012, A\&A, 539, A135

Simoniello, R., Jain, K., Tripathy, S. C., et al. 2013, ApJ, 765, 100

Simoniello, R., Tripathy, S. C., Jain, K., \& Hill, F. 2016, ApJ, 828, 41

Strugarek, A., Beaudoin, P., Charbonneau, P., \& Brun, A. S. 2018, ApJ, 863, 35

Suess, H. E. 1980, Radiocarbon, 22, 200

Thompson, M. J., Christensen-Dalsgaard, J., Miesch, M. S., \& Toomre, J. 2003, ARA\&A, 41, 599

Torrence, C., \& Compo, G. P. 1998, Bull. Am. Meteorol. Soc., 79, 61

Ulrich, R. K., \& Tran, T. 2013, ApJ, 768, 189

Vecchio, A., \& Carbone, V. 2009, A\&A, 502, 981

Vecchio, A., Laurenza, M., Carbone, V., \& Storini, M. 2010, ApJ, 709, L1

Vecchio, A., Laurenza, M., Meduri, D., Carbone, V., \& Storini, M. 2012, ApJ, 749,27

Wang, Y.-M., \& Sheeley, Jr., N. R. 1991, ApJ, 375, 761

Wang, Y.-M., \& Sheeley, Jr., N. R. 2003, ApJ, 590, 1111

Wang, Y.-M., Nash, A. G., \& Sheeley, Jr., N. R. 1989, Science, 245, 712

Wang, Y.-M., Sheeley, Jr., N. R., \& Nash, A. G. 1991, ApJ, 383, 431

Warnecke, J., Rheinhardt, M., Tuomisto, S., et al. 2018, A\&A, 609, A51

Zaqarashvili, T. V., Carbonell, M., Oliver, R., \& Ballester, J. L. 2010, ApJ, 724, L95 


\section{Appendix A: Fourier analyses of SSA and the BL- and turbulent $\alpha$-dynamos}
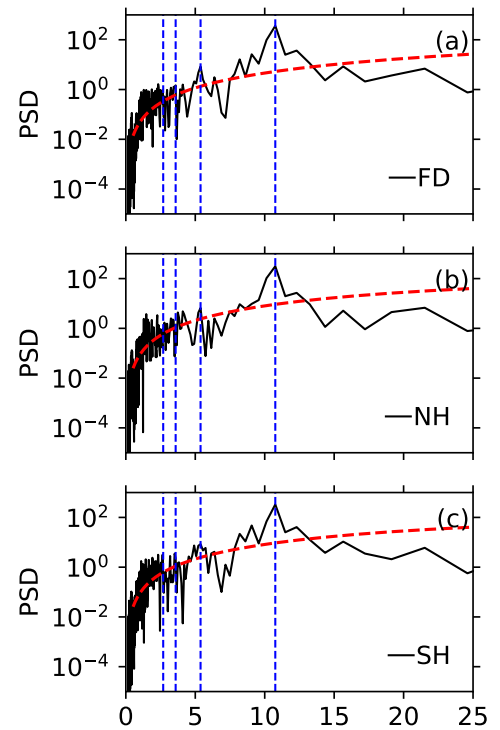

Fig. A.1. Fourier power spectral densities for the SSA data (black lines), lag-1 auto-correlation Fourier power spectral densities (dashedred lines) indicating the significance level of 0.05 , and the highest period and its first three harmonics (dashed-blue lines) for full disk $(a)$, northern hemisphere $(b)$, and southern hemisphere $(c)$.
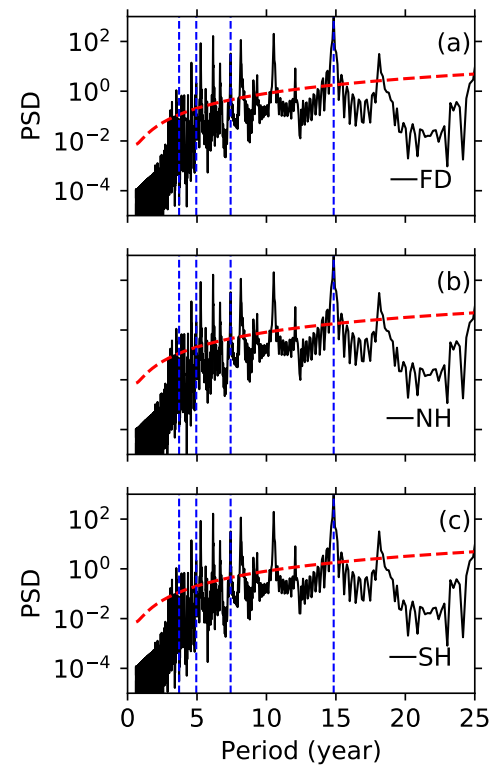

Fig. A.2. Fourier power spectral densities for the BL-dynamo data (black lines), lag-1 auto-correlation Fourier power spectral densities (dashed-red lines) indicating the significance level of 0.05, and the highest period and its first three harmonics (dashed-blue lines) for full disk $(a)$, northern hemisphere $(b)$, and southern hemisphere $(c)$.
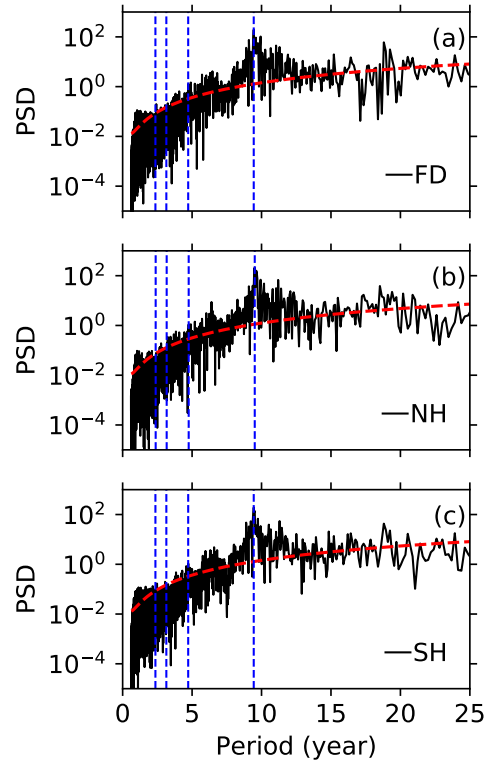

Fig. A.3. Fourier power spectral densities for the turbulent $\alpha$-dynamo data (black lines), lag-1 auto-correlation Fourier power spectral densities (dashed-red lines) indicating the significance level of 0.05 , and the highest period and its first three harmonics (dashed-blue lines) for full disk $(a)$, northern hemisphere $(b)$, and southern hemisphere $(c)$.

\section{Appendix B: Dynamo equations}

The following equations are mostly a reproduction from Rempel (2005a), Kitchatinov \& Rüdiger (2005), Rempel (2006). The basic assumptions made for generation of the differential rotation and meridional circulation in the solar convection zone are as follows.

- Axisymmetry and a spherically symmetric reference state.

- All processes on the convective scale are parameterised; these lead to turbulent viscosity, turbulent heat conductivity, and turbulent angular momentum transport.

- The equations can be linearised under the assumption that $\varrho_{1} \ll \varrho_{0}$ and $p_{1} \ll p_{0}$ and spherically symmetric reference state, where $\varrho_{0}$ and $p_{0}$ are the reference state values and $\varrho_{1}$ and $p_{1}$ are the perturbations caused by the differential rotation. Here we solve axisymmetric hydrodynamic equations that are fully compressible and linearised.

- The entropy equation includes only perturbations from differential rotation and hence the reference state is assumed to be in an energy flux balance. It is also assumed that the effect of convection on entropy perturbations associated with the differential rotation in the convection and overshoot regions is purely diffusive.

- The tachocline at the base of the convection zone is forced by a uniform rotation boundary condition at $r=0.65 R_{\odot}$.

$\frac{\partial \varrho_{1}}{\partial t}=-\frac{1}{r^{2}} \frac{\partial}{\partial r}\left(r^{2} v_{r} \varrho_{0}\right)-\frac{1}{r \sin \theta} \frac{\partial}{\partial \theta}\left(\sin \theta v_{\theta} \varrho_{0}\right)$,

$\frac{\partial v_{r}}{\partial t}=-v_{r} \frac{\partial v_{r}}{\partial r}-\frac{v_{\theta}}{r} \frac{\partial v_{r}}{\partial \theta}+\frac{v_{\theta}^{2}}{r}-\frac{1}{\varrho_{0}}\left[\varrho_{1} g(r)+\frac{\partial p_{1}}{\partial r}\right]$

$+\left(2 \Omega_{0} \Omega_{1}+\Omega_{1}^{2}\right) r \sin ^{2} \theta+\frac{F_{r}}{\varrho_{0}}$, 


$$
\begin{aligned}
\frac{\partial v_{\theta}}{\partial t}= & -v_{r} \frac{\partial v_{\theta}}{\partial r}-\frac{v_{\theta}}{r} \frac{\partial v_{\theta}}{\partial \theta}-\frac{v_{r} v_{\theta}}{r}-\frac{1}{\varrho_{0}} \frac{1}{r} \frac{\partial p_{1}}{\partial \theta} \\
& +\left(2 \Omega_{0} \Omega_{1}+\Omega_{1}^{2}\right) r \sin \theta \cos \theta+\frac{F_{\theta}}{\varrho_{0}} \\
\frac{\partial \Omega_{1}}{\partial t}= & -\frac{v_{r}}{r^{2}} \frac{\partial}{\partial r}\left[r^{2}\left(\Omega_{0}+\Omega_{1}\right)\right] \\
& -\frac{v_{\theta}^{2}}{r \sin ^{2} \theta} \frac{\partial}{\partial \theta}\left[\sin ^{2} \theta\left(\Omega_{0}+\Omega_{1}\right)\right]+\frac{F_{\phi}}{\varrho_{o} r \sin \theta}, \\
\frac{\partial s_{1}}{\partial t}= & -v_{r} \frac{\partial s_{1}}{\partial r}-\frac{v_{\theta}}{r} \frac{\partial s_{1}}{\partial \theta}+v_{r} \frac{\gamma \delta}{H_{p}}+\frac{\gamma-1}{p_{0}} Q \\
& +\frac{1}{\varrho_{0} T_{0}} \operatorname{div}\left(\kappa_{\mathrm{t}} \varrho_{0} T_{0} \nabla s_{1}\right),
\end{aligned}
$$

$E_{r \phi}=E_{\phi r}=r \sin \theta \frac{\partial \Omega_{1}}{\partial r}$,

$E_{\theta \phi}=E_{\phi \theta}=\sin \theta \frac{\partial \Omega_{1}}{\partial r}$,

(B.4) The energy converted by the Reynolds stress is

$Q=\sum_{i, k} \frac{1}{2} E_{i k} R_{i k}$

We transform the entropy equation to an equation of the quantity $\varrho_{0} T_{0} s_{1}$ that better represents the energy perturbation associated with the entropy perturbation to make the importance of the term $\mathrm{Q}$ for a stationary solution more apparent. In the case of a stationary solution, we have $\operatorname{div}\left(\varrho_{0} \boldsymbol{v}\right)=0$, which allows us to rewrite the entropy equation in the following form under the assumption of $|\delta|=\left|\nabla-\nabla_{\text {ad }}\right| \ll 1$,

$p_{1}=p_{0}\left(\gamma \frac{\varrho_{1}}{\varrho_{0}}+s_{1}\right)$

$H_{\mathrm{p}}=\frac{p_{0}}{\varrho_{0} g}$,

$\frac{\mathrm{d} s_{0}}{\mathrm{~d} r}=-\frac{\gamma \delta}{H_{\mathrm{p}}}$.

The last term in Eq. (B.5) describes the turbulent diffusion of entropy perturbation within the convection zone, where $\kappa_{\mathrm{t}}$ represents the turbulent thermal diffusivity.

The viscous force $\boldsymbol{F}$ is given as follows.

$$
\begin{aligned}
F_{r}= & \frac{1}{r^{2}} \frac{\partial}{\partial r}\left(r^{2} R_{r r}\right)+\frac{1}{r \sin \theta} \frac{\partial}{\partial \theta}\left(\sin \theta R_{\theta r}\right) \\
& -\frac{R_{\theta \theta}+R_{\phi \phi}}{r}, \\
F_{\theta}= & \frac{1}{r^{2}} \frac{\partial}{\partial r}\left(r^{2} R_{r \theta}\right)+\frac{1}{r \sin \theta} \frac{\partial}{\partial \theta}\left(\sin \theta R_{\theta \theta}\right) \\
& +\frac{R_{r \theta}-R_{\phi \phi} \cot \theta}{r},
\end{aligned}
$$$$
F_{\phi}=\frac{1}{r^{2}} \frac{\partial}{\partial r}\left(r^{2} R_{r \phi}\right)+\frac{1}{r \sin \theta} \frac{\partial}{\partial \theta}\left(\sin \theta R_{\theta \phi}\right)
$$$$
+\frac{R_{r \phi}+R_{\theta \phi} \cot \theta}{r}
$$

with the Reynolds stress tensor

$R_{i k}=-\varrho_{0}\left\langle v_{i}^{\prime} v_{k}^{\prime}\right\rangle=v_{\mathrm{t}} \varrho_{0}\left(E_{i k}-\frac{2}{3} \delta_{i k} \operatorname{div} \boldsymbol{v}+\Lambda_{i k}\right)$,

where $\Lambda_{i k}$ is the non-diffusive Reynolds stress and the deformation tensor, $E_{i k}=v_{i ; k}+v_{k ; i}$, is given for the spherical coordinates as follows.

$$
\begin{aligned}
& E_{r r}=2 \frac{\partial v_{r}}{\partial r}, \\
& E_{\theta \theta}=2 \frac{1}{r} \frac{\partial v_{\theta}}{\partial \theta}+2 \frac{v_{r}}{r}, \\
& E_{\phi \phi}=\frac{2}{r}\left(v_{r}+v_{\theta} \cot \theta\right), \\
& E_{r \theta}=E_{\theta r}=r \frac{\partial}{\partial r} \frac{v_{\theta}}{r}+\frac{1}{r} \frac{\partial v_{r}}{\partial \theta},
\end{aligned}
$$

$$
\begin{aligned}
\operatorname{div}\left(\boldsymbol{v} \varrho_{0} T_{0} s_{1}-\kappa_{t} \varrho_{0} T_{0} \operatorname{grad} s_{1}\right)= & (\gamma-1)\left(\frac{\varrho_{0} T_{0}}{p_{0}} Q-v_{r} \frac{\varrho_{0} T_{0} s_{1}}{\gamma H_{\mathrm{p}}}\right) \\
& +v_{r} \gamma \delta \frac{\varrho_{0} T_{0}}{H_{\mathrm{p}}} .
\end{aligned}
$$

For the background $\varrho_{0}, p_{0}$, and $T_{0}$, we use an adiabatic hydrostatic stratification, assuming an $\sim r^{2}$ dependence of the gravitational acceleration given by

$T_{0}(r)=T_{\mathrm{bc}}\left[1+\frac{\gamma-1}{\gamma} \frac{r_{\mathrm{bc}}}{H_{\mathrm{bc}}}\left(\frac{r_{\mathrm{bc}}}{r}-1\right)\right]$,

$p_{0}(r)=p_{\mathrm{bc}}\left[1+\frac{\gamma-1}{\gamma} \frac{r_{\mathrm{bc}}}{H_{\mathrm{bc}}}\left(\frac{r_{\mathrm{bc}}}{r}-1\right)\right]^{\gamma /(\gamma-1)}$,

$\varrho_{0}(r)=\varrho_{\mathrm{bc}}\left[1+\frac{\gamma-1}{\gamma} \frac{r_{\mathrm{bc}}}{H_{\mathrm{bc}}}\left(\frac{r_{\mathrm{bc}}}{r}-1\right)\right]^{1 /(\gamma-1)}$,

$g(r)=g_{\mathrm{bc}}\left({\frac{r}{r_{\mathrm{bc}}}}^{-2}\right)$,

where $T_{\mathrm{bc}}, \varrho_{\mathrm{bc}}$, and $p_{\mathrm{bc}}$ represent temperature, density, and pressure values at the base of the convection zone, $r=r_{\mathrm{bc}}$. The term $H_{\mathrm{bc}}=p_{\mathrm{bc}} /\left(\varrho_{\mathrm{bc}} g_{\mathrm{bc}}\right)$ is the pressure scale height and $g_{\mathrm{bc}}$ is the value of the gravity at $r_{\mathrm{bc}}$. In our simulations, we use $r_{\mathrm{bc}}=$ $0.71 R_{\odot}, p_{\mathrm{bc}}=6 \times 10^{12} \mathrm{~Pa}, \varrho_{\mathrm{bc}}=200 \mathrm{~kg} \mathrm{~m}^{-3}, g_{\mathrm{bc}}=520 \mathrm{~m} \mathrm{~s}^{-2}$, and $R_{\odot}=7 \times 10^{8} \mathrm{~km}$.

For the superadiabaticity $\delta$ we assume the following profile:

$\delta=\delta_{\text {conv }}+\frac{1}{2}\left(\delta_{\mathrm{os}}-\delta_{\text {conv }}\right)\left[1-\tanh \left(\frac{r-r_{\text {tran }}}{d_{\text {tran }}}\right)\right]$,

$\delta_{\mathrm{conv}}=\delta_{\mathrm{top}} \exp \left(\frac{r-r_{\mathrm{max}}}{d_{\mathrm{top}}}\right)+\delta_{\mathrm{cz}} \frac{r-r_{\mathrm{sub}}}{r_{\mathrm{max}}-r_{\mathrm{sub}}}$

where $\delta_{\text {top }}, \delta_{\mathrm{cz}}$, and $\delta_{\mathrm{os}}$ represent the values of superadiabaticity at the top of the domain $\left(r=r_{\max }\right)$, in the bulk of the convection zone, and in the overshoot region. The terms $r_{\text {sub }}$ and $r_{\text {tran }}$ denote the radius at which the stratification within the convection zone turns weakly subadiabatic and the radius of transition toward stronger subadiabatic stratification, respectively. The steepness of the transition towards larger superadiabaticities at the top of the domain and towards the overshoot regions are determined by $d_{\text {top }}$ and $d_{\text {tran }}$. 
We assume that the diffusivities only depend on the radial coordinate and turbulent viscosity and thermal conductivity are given as

$v_{\mathrm{t}}=\frac{v_{0}}{2}\left[1+\tanh \left(\frac{r-r_{\text {tran }}+\Delta}{d_{\kappa v}}\right)\right] f_{\mathrm{c}}(r)$,

$\kappa_{\mathrm{t}}=\frac{\kappa_{0}}{2}\left[1+\tanh \left(\frac{r-r_{\text {tran }}+\Delta}{d_{\kappa v}}\right)\right] f_{\mathrm{c}}(r)$

$f_{\mathrm{c}}(r)=\frac{1}{2}\left[1+\tanh \left(\frac{r-r_{\mathrm{bc}}}{d_{\mathrm{bc}}}\right)\right]$

$\Delta=d_{\kappa \nu} \tanh ^{-1}\left(2 \alpha_{\kappa \nu}-1\right)$.

The radial and latitudinal angular momentum fluxes are proportional to the off-diagonal components of the correlation tensor in spherical coordinates, $Q_{r \phi}$ and $Q_{\theta \phi}$ and they are finite and parametrised as

$Q_{r \phi}^{\Lambda}=v_{t} \Omega V \sin \theta$,

$Q_{\theta \phi}^{\Lambda}=v_{t} \Omega H \cos \theta$,

where $V$ and $H$ are the normalised vertical and horizontal fluxes $V=V^{(0)}\left(\Omega^{*}\right)-H^{(1)}\left(\Omega^{*}\right) \cos ^{2} \theta$,

$H=H^{(1)}\left(\Omega^{*}\right) \sin ^{2} \theta$,

$V^{(0)}=\left(\frac{\ell_{\text {corr }}}{H_{\rho}}\right)^{1 / 2}\left(J_{0}\left(\Omega^{*}\right)+a I_{0}\left(\Omega^{*}\right)\right)$,

$H^{(1)}=\left(\frac{\ell_{\text {corr }}}{H_{\rho}}\right)^{1 / 2}\left(J_{1}\left(\Omega^{*}\right)+a I_{1}\left(\Omega^{*}\right)\right)$,

with

$$
\begin{aligned}
J_{0}\left(\Omega^{*}\right)= & \frac{1}{2 \Omega^{* 4}}\left(9-\frac{2 \Omega^{* 2}}{1+\Omega^{* 2}}-\frac{\Omega^{* 2}+9}{\Omega^{*}} \arctan \Omega^{*}\right), \\
J_{1}\left(\Omega^{*}\right)= & \frac{1}{2 \Omega^{* 4}}\left(45+\Omega^{* 2}-\frac{4 \Omega^{* 2}}{1+\Omega^{* 2}}\right. \\
& \left.+\frac{\Omega^{* 4}-12 \Omega^{* 2}-45}{\Omega^{*}} \arctan \Omega^{*}\right),
\end{aligned}
$$

and

$$
\begin{aligned}
I_{0}\left(\Omega^{*}\right)= & \frac{1}{4 \Omega^{* 4}} \\
& \times\left(-19-\frac{5}{1+\Omega^{* 2}}+\frac{3 \Omega^{* 2}+24}{\Omega^{*}} \arctan \Omega^{*}\right), \\
I_{1}\left(\Omega^{*}\right)= & \frac{3}{4 \Omega^{* 4}} \\
& \times\left(-15-\frac{2 \Omega^{* 2}}{1+\Omega^{* 2}}+\frac{3 \Omega^{* 2}+15}{\Omega^{*}} \arctan \Omega^{*}\right),
\end{aligned}
$$

where $\Omega^{*}$ denotes the Coriolis number.

The mean field differential rotation and meridional circulation model described above is then coupled with the axisymmetric mean field dynamo equations. The computed magnetic field is allowed to feed back on differential rotation and meridional circulation via the Lorentz force. The vector potential is introduced in the induction equation to satisfy the constrain $\nabla \cdot \boldsymbol{B}=0$. The coupled equations are

$$
\begin{aligned}
\frac{\partial v_{r}}{\partial t}= & -v_{r} \frac{\partial v_{r}}{\partial r}-\frac{v_{\theta}}{r} \frac{\partial v_{r}}{\partial \theta}+\frac{v_{\theta}^{2}}{r}-\frac{\partial}{\partial r} \frac{p_{\mathrm{tot}}}{\varrho_{0}}+\frac{p_{\mathrm{mag}}}{\gamma p_{0}} g \\
& +\frac{s_{1}}{\gamma} g+\left(2 \Omega_{0} \Omega_{1}+\Omega_{1}^{2}\right) r \sin ^{2} \theta+\frac{1}{\varrho_{0}}\left(F_{r}^{v}+F_{r}^{B}\right), \\
\frac{\partial v_{\theta}}{\partial t}= & -v_{r} \frac{\partial v_{\theta}}{\partial r}-\frac{v_{\theta}}{r} \frac{\partial v_{\theta}}{\partial \theta}-\frac{v_{r} v_{\theta}}{r}-\frac{1}{r} \frac{\partial}{\partial \theta} \frac{p_{\mathrm{tot}}}{\varrho_{0}} \\
& +\left(2 \Omega_{0} \Omega_{1}+\Omega_{1}^{2}\right) r \sin \theta \cos \theta+\frac{1}{\varrho_{0}}\left(F_{\theta}^{v}+F_{\theta}^{B}\right),
\end{aligned}
$$

$$
\begin{aligned}
\frac{\partial \Omega_{1}}{\partial t}= & -\frac{v_{r}}{r^{2}} \frac{\partial}{\partial r}\left[r^{2}\left(\Omega_{0}+\Omega_{1}\right)\right] \\
& -\frac{v_{\theta}^{2}}{r \sin ^{2} \theta} \frac{\partial}{\partial \theta}\left[\sin ^{2} \theta\left(\Omega_{0}+\Omega_{1}\right)\right] \\
& +\frac{1}{\varrho_{o} r \sin \theta}\left(F_{\phi}^{v}+F_{\phi}^{B}\right),
\end{aligned}
$$

$\frac{\partial s_{1}}{\partial t}=-v_{r} \frac{\partial s_{1}}{\partial r}-\frac{v_{\theta}}{r} \frac{\partial s_{1}}{\partial \theta}+v_{r} \frac{\gamma \delta}{H_{\mathrm{p}}}+\frac{\gamma-1}{p_{0}} Q$

$$
+\frac{1}{\varrho_{0} T_{0}} \nabla \cdot\left(\kappa_{\mathrm{t}} \varrho_{0} T_{0} \nabla s_{1}\right)+\frac{\gamma-1}{p_{0}} \eta_{\mathrm{t}}(\nabla \times \boldsymbol{B})^{2},
$$

$\frac{\partial B_{\phi}}{\partial t}=-\frac{1}{r} \frac{\partial}{\partial r}\left(r v_{r} B_{\phi}\right)-\frac{1}{r} \frac{\partial}{\partial \theta}\left(v_{\theta} B_{\phi}\right)+r \sin \theta B_{r} \frac{\partial \Omega_{1}}{\partial r}$

$$
\begin{aligned}
& +\sin \theta B_{\theta} \frac{\partial \Omega_{1}}{\partial \theta}+\eta_{\mathrm{t}}\left[\Delta-\frac{1}{(r \sin \theta)^{2}}\right] B_{\phi} \\
& +\frac{1}{r} \frac{\partial \eta_{\mathrm{t}}}{\partial r} \frac{\partial}{\partial r}\left(r B_{\phi}\right)+\frac{1}{r^{2}} \frac{\partial \eta_{\mathrm{t}}}{\partial \theta} \frac{1}{\sin \theta} \frac{\partial}{\partial \theta}\left(\sin \theta B_{\phi}\right),
\end{aligned}
$$

$$
\begin{aligned}
\frac{\partial A}{\partial t}= & -\frac{v_{r}}{r} \frac{\partial}{\partial r}(r A)-\frac{v_{\theta}}{r \sin \theta} \frac{\partial}{\partial \theta}(\sin \theta A) \\
& +\eta_{\mathrm{t}}\left[\Delta-\frac{1}{(r \sin \theta)^{2}}\right] A+S\left(r, \theta, B_{\phi}\right) .
\end{aligned}
$$

In the above equations, $\eta_{\mathrm{t}}$ denotes turbulent magnetic diffusivity, which is given as

$\eta_{\mathrm{t}}=\eta_{\mathrm{c}}+f_{\mathrm{c}}(r)\left[\eta_{\mathrm{bc}}-\eta_{\mathrm{c}}+f_{\mathrm{cz}}(r)\left(\eta_{\mathrm{cz}}-\eta_{\mathrm{bc}}\right)\right]$,

$f_{\mathrm{cz}}(r)=\frac{1}{2}\left[1+\tanh \left(\frac{r-r_{\mathrm{cz}}}{d_{\mathrm{cz}}}\right)\right]$,

$f_{\mathrm{c}}(r)=\frac{1}{2}\left[1+\tanh \left(\frac{r-r_{\mathrm{bc}}}{d_{\mathrm{bc}}}\right)\right]$.

The source term $S\left(r, \theta, B_{\phi}\right)$ in the induction equation is given as

$S\left(r, \theta, B_{\phi}\right)=\alpha_{0} \bar{B}_{\phi, b c}(\theta) f_{\alpha}(r) g_{\alpha}(\theta)$,

where

$\bar{B}_{\phi, \mathrm{bc}}(\theta)=\int_{r_{\min }}^{r_{\max }} \mathrm{d} r h(r) B_{\phi}(r, \theta)$. 
F. Inceoglu et al.: Constraining non-linear dynamos using QBOs

For the non-local BL-effect:

$f_{\alpha}(r)=\max \left[0,1-\frac{\left(r-r_{\max }\right)^{2}}{d_{\alpha}^{2}}\right]$,

$g_{\alpha}(r)=\frac{(\sin \theta)^{4} \cos \theta}{\max \left[(\sin \theta)^{4} \cos \theta\right]}$.
As for the turbulent $\alpha$-dynamo operating in the bottom half of the convection zone, the source term becomes

$f_{\alpha}(r)=\frac{1}{4}\left[1+\operatorname{erf}\left(\frac{r-r_{2}}{d_{2}}\right)\right]\left[1-\operatorname{erf}\left(\frac{r-r_{3}}{d_{3}}\right)\right]$,

$g_{\alpha}(r)=\frac{\sin \theta \cos \theta}{\max [\sin \theta \cos \theta]}$ 